\title{
Studies of MaPMTs with beetle-chip read-out
}

\author{
F. Muheim \\ School of Physics, University of Edinburgh, Mayfield Road, Edinburgh, EH9 3JZ, Scotland/UK \\ Available online 13 September 2005 \\ On behalf of the LHCb RICH MaPMT Group
}

\begin{abstract}
We have evaluated the 64-channel Multianode Photo-Multiplier (MaPMT) with 8-stage dynodes for the LHCb RICH detectors. With a Beetle 1.2 chip to read-out the MaPMT, we have demonstrated that the MaPMT performance is as expected using particle beams and LED light sources. We have also measured the pulse shape from 12-stage dynode MaPMTs, read out with the Beetle 1.2-MA0 chip.
\end{abstract}

(C) 2005 Elsevier B.V. All rights reserved.

PACS: $85.60 . \mathrm{H} ; 29.40 . \mathrm{K}$

Keywords: Multianode photo multiplier tubes; Ring Imaging Cherenkov detectors; LHCb

\section{Overview}

We report on studies of Multi-anode PhotoMultiplier Tubes (MaPMTs) as photo-detectors for Ring Imaging Cherenkov (RICH) counters. The 64-channel MaPMTs were evaluated as an option for the $\mathrm{RICH}$ detectors of the $\mathrm{LHCb}$ experiment which will operate at the Large Hadron Collider (LHC). Earlier results on 12dynode stage MaPMTs have been presented in Refs. [1,2]. Here we present new measurements with 8 and 12-dynode stage MaPMTs with recently developed "Beetle" read-out electronics.

E-mail address: f.muheim@ed.ac.uk.
In the next Section we describe the properties of the different types of MaPMTs and the Beetle-chip electronics. Measurements with an LED light source are reported in Section 3. The main set of the measurements were taken with particle beams at the CERN Proton Synchrotron (PS) using MaPMTs with 8 and 12-dynode stages and are presented in Sections 4 and 5, respectively. In the final section we give conclusions.

\section{Multi-anode photo-multiplier tubes and beetle- chip read-out}

The 64-channel MaPMT contains an $8 \times 8$ array of dynode chains within a single 1-in. 
vacuum envelope. The effective pixel size of the 64-channel MaPMT is $2.1 \mathrm{~mm}$. The MaPMTs have UV-glass windows and a bi-alkali photocathode. The peak quantum efficiency quoted by the vendor is $25 \%$ at $\lambda=360 \mathrm{~nm}$. The $\mathrm{R} 7600$ MaPMT with 12-stage dynode chains has a gain of about 300,000 with the photo-cathode set at a voltage of $-800 \mathrm{~V}$.

The read-out electronics for the $\mathrm{LHCb} \mathrm{RICH}$ detectors must be able to run at the LHC interaction rate of $40 \mathrm{MHz}$. A 64-channel MaPMT with 8 dynode stages became available two years ago. Initial tests confirmed that the tube has a gain of about 50,000 at $-800 \mathrm{~V}$. This gain is well matched to electronics developed for silicon detectors at LHC speed. The Beetle 1.2 read-out chip has 128 channels, low noise and a dynamic range of 10 photo-electrons $[3,4]$.

In order to read out the 8-dynode stage MaPMT with the Beetle 1.2 chip we designed a dedicated multi-layer printed circuit motherboard containing one Beetle 1.2 chip. Each motherboard has two pin-grid array sockets which can be attached to two MaPMTs by means of a bleeder-board which also supplies the high voltage to the dynode chain. The width of the card is slightly narrower than the pitch of close-packed MaPMTs and the read-out of a $3 \times 3$ array of MaPMTs described below required six motherboards. The 128 input signal lines from a pair of MaPMTs are connected to the Beetle chip by means of a pitch adaptor on the motherboard. The Beetle chip read-out is multiplexed, either 128 or 32 -fold. To transmit the analogue signal over a distance of a few metres the four signal lines are amplified with differential amplifier chips mounted on the motherboard. The signals are then transmitted to and digitised by the "Front End Driver" (FED) [5]. In addition, each motherboard feeds the supply voltages, the control $\left(\mathrm{I}^{2} \mathrm{C}\right)$ signals, the low-voltage differential signalling (LVDS) trigger and the $40 \mathrm{MHz}$ clock signals to the chip. A simple test-board which connected at most 12 MaPMT pixels was also available.

We also designed a customised read-out chip, the Beetle 1.2-MA0 chip [6], with an amplification and a dynamic range matched to the gain of a 12-dynode stage MaPMT. Modifications to the standard Beetle 1.2 chip were made to the front- end and to the shaper amplifier; the digital parts and multiplexing pipeline of the chip were left unaltered. For practical reasons only 64 input channels were equipped with the MA0 design, the remaining channels were either equipped with a charge divider or used as test channels. The Beetle 1.2-MA0 chip was produced in early 2003 and measurements with MaPMTs are reported here. To connect to the MaPMTs, the motherboard was equipped without modifications with the Beetle 1.2-MA0 chip. Both the standard Beetle 1.2 and the Beetle 1.2-MA0 chips were read out in analogue mode.

\section{Scans with an LED}

We have measured the pulse height spectra of an 8-dynode and 12-dynode stage MaPMT by illuminating the tubes with short LED pulses. For these studies we used the simple test-board which allowed us to connect up to 12 MaPMT channels and which could be equipped with either a Beetle 1.2 or a Beetle 1.2-MA0 chip, respectively. Fig. 1 shows the pulse height spectra for a single pixel of an 8-dynode and a 12-dynode stage MaPMT, respectively. Both of these plots show clear separations between the pedestals and the (mostly) single photo-electron signals. It is also apparent that the separation for the 12-dynode stage MaPMT is better than for the 8-dynode stage MaPMT. As expected from simulation, the noise performance of the Beetle 1.2-MA0 is excellent. Varying the high voltage at the photocathode shows that an increase of $50 \mathrm{~V}$ corresponds to a doubling of the average pulse height.

\section{Performance of the 8-dynode stage MaPMT}

Particle beams generate Cherenkov light at a well defined rate and give direct measurements of the performance of a RICH counter. We used a beam of negative particles $(95 \%$ pions and $5 \%$ electrons) with a momentum of $10 \mathrm{GeV} / c$ from the CERN PS beam. Nine 8-dynode stage MaPMTs were mounted in a $3 \times 3$ cluster onto the focal plane of a RICH prototype vessel [1]. A $100 \mathrm{~cm}$ 

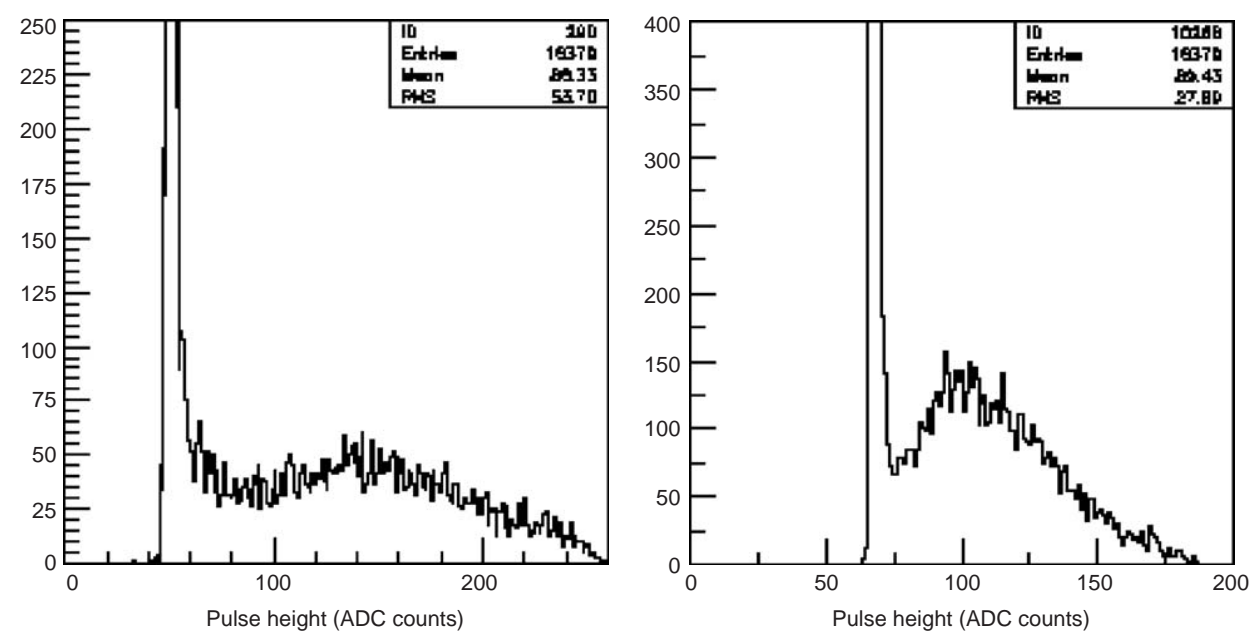

Fig. 1. Pulse height spectrum of a 8-dynode stage MaPMT pixel (left) with a high voltage $-850 \mathrm{~V}$ and of a 12-dynode stage MaPMT pixel (right) at $-900 \mathrm{~V}$.

long gaseous $\mathrm{CF}_{4}$ radiator at a pressure of 800 mbar produced Cherenkov photons which, by means of a spherical mirror with focal length $1117 \mathrm{~mm}$, were imaged onto a ring on the MaPMT cluster.

The active area of an MaPMT is only $38 \%$ when accounting for the rims and the inactive $0.2 \mathrm{~mm}$ gaps between pixels. Therefore the full geometrical coverage, $85 \%$ active area, was recovered by mounting fused silica lenses with one spherical and one flat surface in front of each MaPMT. The lens demagnification increases the effective pixel size to $3.2 \mathrm{~mm}$.

A full GEANT4 Monte Carlo simulation of this set-up has been carried out. The beam divergence as measured with a silicon telescope was included. The Cherenkov photons were generated in the radiator and traced optically via mirror, quartz window and lenses to the MaPMT photo-cathode. Photo-electrons and MaPMT pixel hits were then created according to the quantum efficiency. The simulation predicts that an average yield of 6.2 photo-electrons, i.e. hits per event, should be measured assuming no signal loss correction.

The event trigger was provided with a coincidence of four scintillators in the beam line. Runs were recorded with the photo-cathode voltage set at values from -750 to $-1000 \mathrm{~V}$. In Fig. 2 (left) we show a typical single event at $-900 \mathrm{~V}$. A hit or photo-electron is defined as a pixel with a pulse height larger than $5 \sigma$ above pedestal, where $\sigma$ is the Gaussian pedestal width. Fig. 2 (right) shows the integrated number of hits of a run of $16.4 \mathrm{k}$ events. A Cherenkov ring is clearly visible. The yield is 11.2 photo-electrons per event; a significant fraction of these hits are caused by cross-talk as is illustrated by the double hits observed in the left hand figure. The main cross-talk is an asymmetric component of $13 \%(4 \%)$ of the signal amplitude into the next (previous) channel of the Beetle readout pipeline location. In addition, there is a smaller symmetric cross-talk between neighbouring channels on the input lines. The cross-talk is removed by producing a map of cross-talk partners for each pixel and by re-distributing the cross-talk fraction of the measured pulse height for hits with a larger pulse height into a partner pixel and subtracting the corresponding pulse height. This procedure produces a $4 \%$ over-correction as genuine double photon hits can also be treated as cross-talk. In Fig. 3 (left) we show the same data after applying the cross-talk correction. The horizontal width of the Cherenkov ring is visibly reduced. In Fig. 3 (right) the photon yield is shown as a function of MaPMT high voltage before and after cross-talk correction. At $-900 \mathrm{~V}$ a corrected yield of 6.5 photo-electrons is measured, which is in good agreement with the simulation. At $-900 \mathrm{~V}$, the 

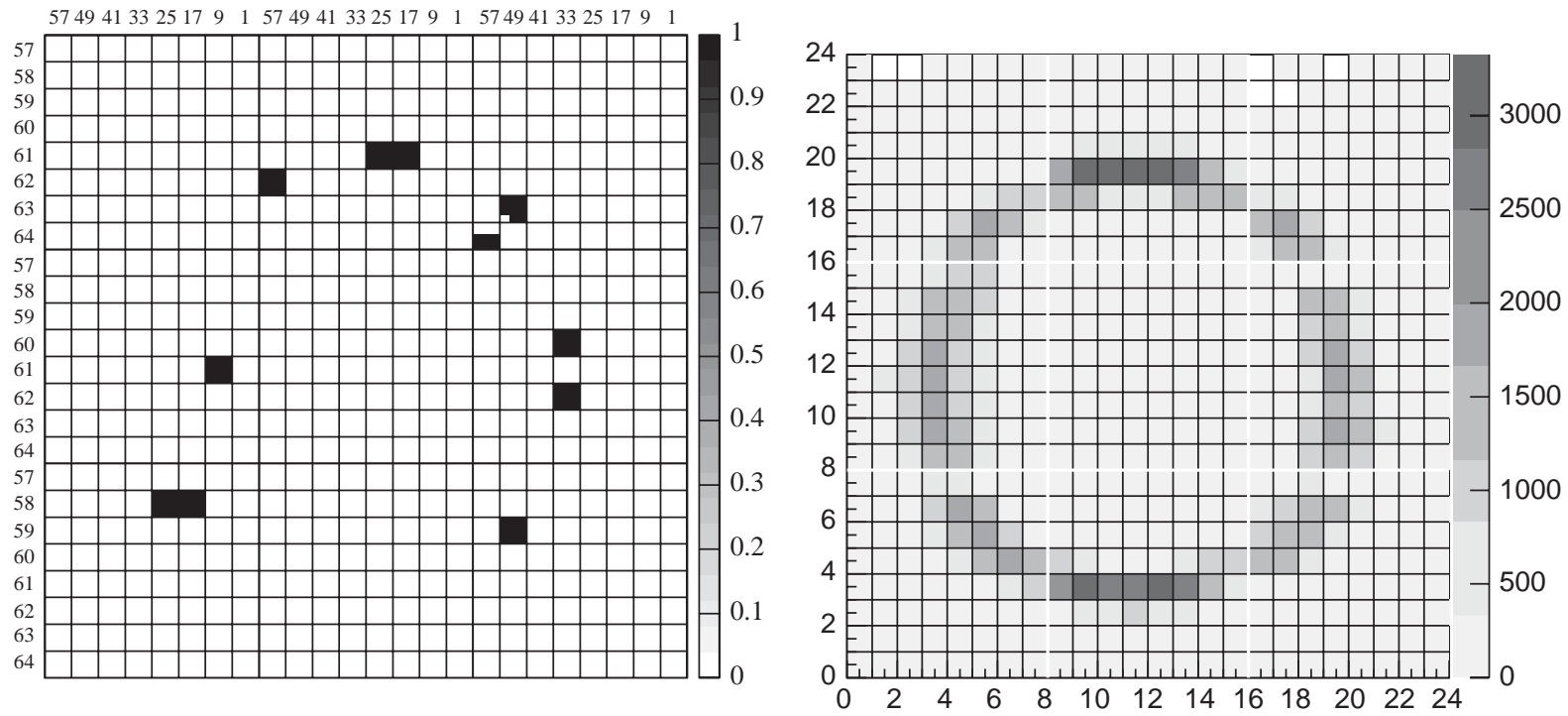

Fig. 2. A typical signal event (left) and integrated raw data hits (right) at $-900 \mathrm{~V}$.
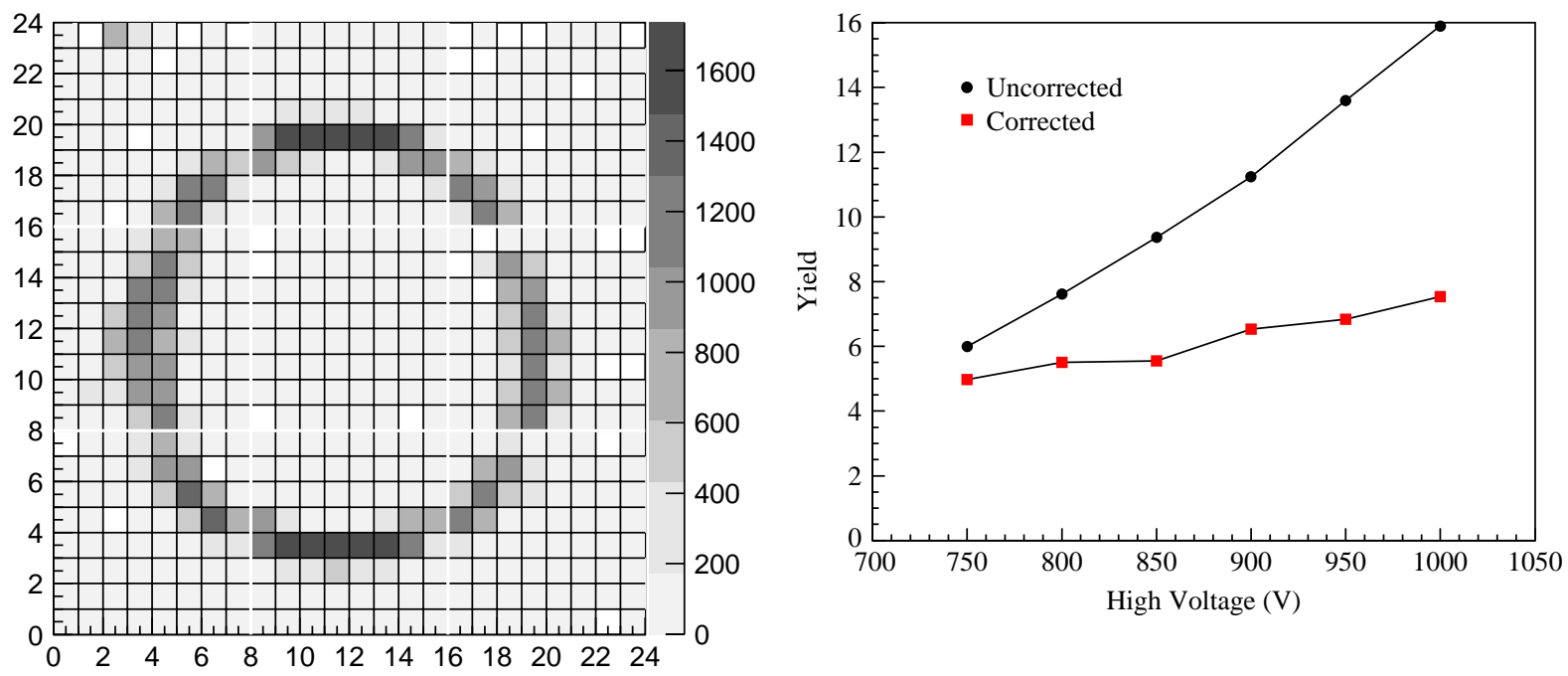

Fig. 3. Photo-electron hits at $-900 \mathrm{~V}$ (left). Photon yield versus high voltage (right).

signal loss below the threshold cut was estimated to be about $7 \%$. The measured performance of the 8-dynode stage MaPMT is also in agreement with previous results using 12-dynode stage MaPMTs [1] and fulfils the requirements for the $\mathrm{LHCb}$ RICH requirements. All the results presented above are preliminary.

\section{Beetle 1.2-MA0 pulse shape}

We also equipped the RICH vessel with a $3 \times 2$ array of 12-dynode stage MaPMTs which were connected to three motherboards with Beetle 1.2MA0 chips. Only half of the MaPMT channels, corresponding to a partial Cherenkov ring, were 
read out with input attenuator channels. We observed a photo-electron yield which was roughly in agreement with expectations. Cross-talk was visible at the same level as for the Beetle 1.2 and was removed.

The MaPMT pulse height is recorded by the Beetle 1.2-MA0 every $25 \mathrm{~ns}$, allowing a number of samples over the pulse profile. The samples are distributed in time randomly with respect to the asynchronous trigger. The time difference between the trigger and the next $25 \mathrm{~ns}$ clock cycle was recorded with a TDC. This allowed a measurement of the pulse height spectra as a function of time. The individual pulse height spectra were fit in $5 \mathrm{~ns}$ time bins. All fit parameters except the mean pulse height were fixed by the results of the fit in the time window where the pulse heights were largest. In Fig. 4 we show the distribution of mean pulse height as a function of the time. This is a measure of the Beetle 1.2MA0 pulse shape. We find good agreement with simulation.

\section{Conclusions}

We have successfully tested a close-packed $3 \times 3$ array of 8-dynode stage MaPMTs read-out with the Beetle 1.2 chip. The photo-electron yield is as expected and fulfils the $\mathrm{LHCb} \mathrm{RICH}$ requirements. Electronic cross-talk is large and needs to be addressed. In addition, we have shown that the Beetle 1.2-MA0 chip which was specifically designed to read-out the 12-dynode stage MaPMT works according to specifications.

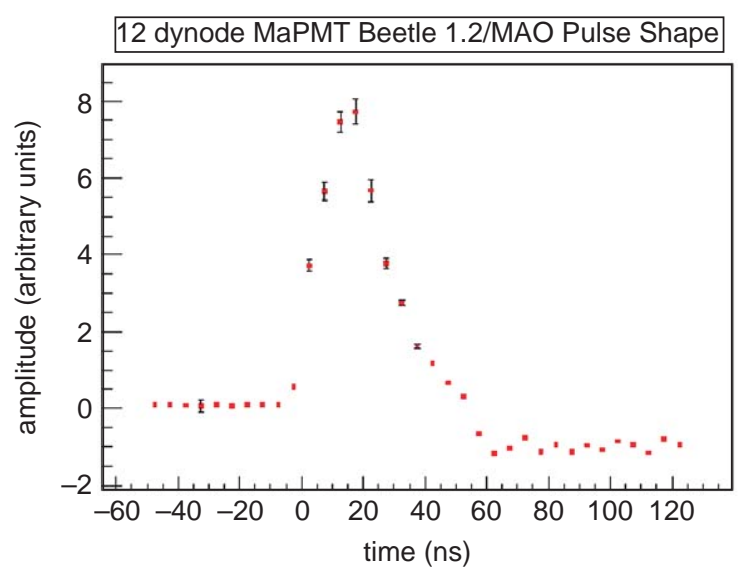

Fig. 4. Mean pulse height versus time for the Beetle 1.2-MA0.

I thank all my colleagues from the $\mathrm{LHCb} \mathrm{RICH}$ MaPMT group for their efforts to produce the test beam data. In addition, I thank Reinhardt Chamonal, Neville Harnew and Andrew Pickford for help in preparing this presentation.

\section{References}

[1] E. Albrecht, et al., Nucl. Instr. and Meth. A 488 (2002) 110.

[2] F. Muheim, Nucl. Instr. and Meth. A 502 (2003) 52.

[3] N. Van Bakel, et al., The Beetle Reference Manual, v1.0, LHCb 2001-046, Electronics, 2001.

[4] N. Van Bakel, et al., Nucl. Instr. and Meth. A 509 (2003) 176.

[5] S.A. Baird, et al., Snowmass 1999, Electronics for LHC Experiments, CERN/LHCC/98-33 (1999) 357.

[6] N. Smale, D.Phil Thesis, University of Oxford, November 2004. 